\title{
High-sensitivity observations of solar flare decimeter radiation
}

\author{
A. O. Benz, P. Messmer, and C. Monstein \\ Institute of Astronomy, ETH-Zentrum, 8092 Zurich, Switzerland \\ Received 25 May 2000 / Accepted 7 November 2000

\begin{abstract}
A new acousto-optic radio spectrometer has observed the $1-2 \mathrm{GHz}$ radio emission of solar flares with unprecedented sensitivity. The number of detected decimeter type III bursts is greatly enhanced compared to observations by conventional spectrometers observing only one frequency at the time. The observations indicate a large number of electron beams propagating in dense plasmas. For the first time, we report weak, reversed drifting type III bursts at frequencies above simultaneous narrowband decimeter spikes. The type III bursts are reliable signatures of electron beams propagating downward in the corona, apparently away from the source of the spikes. The observations contradict the most popular spike model that places the spike sources at the footpoints of loops. Conspicuous also was an apparent bidirectional type U burst forming a fish-like pattern. It occurs simultaneously with an intense U-burst at $600-370 \mathrm{MHz}$ observed in Tremsdorf. We suggest that it intermodulated with strong terrestrial interference (cellular phones) causing a spurious symmetric pattern in the spectrogram at $1.4 \mathrm{GHz}$. Symmetric features in the 1-2 GHz range, some already reported in the literature, therefore must be considered with utmost caution.
\end{abstract}

Key words. acceleration of particles - Sun: corona - Sun: flares - Sun: magnetic fields - Sun: radio radiation

\section{Introduction}

The acceleration of a large population of superthermal electrons is a characteristic of solar flares. The acceleration process is still unclear, although the original energy release is generally, but not unanimously believed to be due to reconnection (e.g. reviews by Tandberg-Hanssen \& Emslie 1988; Priest \& Forbes 2000). Most of these particles escape from the acceleration region along magnetic field lines. If the distance of propagation is long enough, a bump-on-tail instability develops driving Langmuir waves. Thus one may expect plenty of radio emission of type III in every flare. This is not the case as pointed out e.g. by Simnett \& Benz (1986). There may be several reasons conspiring: (i) Observations were made mostly below $1 \mathrm{GHz}$ corresponding to densities below a few times $10^{9} \mathrm{~cm}^{-3}$, as was the case in the above mentioned study and in earlier work. However, flare particle acceleration and propagation is suspected to generally occur at higher densities (Miller et at. 1997). (ii) Even harmonic radio emission (at twice the plasma frequency) is increasingly free-free absorbed at higher frequencies, thus the observed type III bursts appear weaker. (iii) The thermal background emission of the Sun increases strongly with frequency around $1 \mathrm{GHz}$, making burst detection even more difficult.

Send offprint requests to: A. O. Benz,

e-mail: benz@astro.phys.ethz.ch
Magnetic fields at low coronal altitude and particularly in active regions are well known to have predominantly loop-like shapes. Thus electron beams cannot easily escape into interplanetary space. Electron beams radiating type III bursts at decimeter frequencies must follow such loops and thus have a short total lifetime. Due to the smallness of the loops and small density range, they have often a small bandwidth (Benz et al. 1983). Also, the instantaneous duration of bursts decreases with frequency about in inverse proportion (Staehli \& Benz 1987; Meléndez et al. 1999). Electron beams moving into the direction of higher magnetic field and density emit less radio emission (Robinson \& Benz 2000). Small bandwidth, short duration, and downward motion enhance the difficulty for observations even further.

On the other hand, the observation of high-frequency type III bursts is very attractive as electron beams following magnetic field lines map the magnetic geometry into the frequency-time plane (i.e. the spectrogram). Decimeter type III bursts thus outline magnetic fields related to reconnection.

Meterwave type III bursts have been reported to be associated by narrowband spikes above the starting frequencies in $10 \%$ of all cases (Benz et al. 1983). These type III emissions are regular drifting bursts, representing electron beams propagating toward lower density, i.e. upward in the corona. Groups of meterwave spikes are well correlated with individual type III bursts (Benz et al. 1996). 
Metric spikes usually occur in a relatively small band of frequencies around $300 \mathrm{MHz}$. Upward drifting type III bursts have also been noted in practically all events of decimeter spikes (Benz \& Güdel 1989). Such associated type III bursts occur in the meter waves, presumably at higher altitude and are often poorly correlated with time structures of the spike group. Narrowband spikes at meter and decimeter wavelength have similar properties concerning individual bursts, including duration, bandwidth and polarization (review by Benz 1986). However, decimeter spikes form much richer groups extending over a wider frequency band and are well associated with hard X-ray events (Benz \& Kane 1986; Güdel et al. 1989).

Reversed, downward drifting type III bursts have never been noted in association with any spikes. This seems to suggest that the sources of spike emission are located close to the bottom of the corona, near the region where energetic electrons are lost from the trap. It has generally been taken as evidence for spikes to occur at the footpoints of loops, where the velocity distribution of trapped electrons has a loss-cone distribution and is unstable toward the generally favored growing upper hybrid, Bernstein or electron cyclotron waves (Melrose \& Dulk 1982; Sharma et al. 1982; cf. recent theoretical work by Conway \& Willes 2000). In the alternative scenario, on the other hand, suggesting that spikes are a direct signature of the acceleration process and their sources therefore at some finite altitude, it is not clear why reversed drifting type III bursts indicating downward drifting electron beams have never been observed.

Here we report on observations with a multichannel spectrometer far exceeding the sensitivity of the usual frequency-agile instruments. We concentrate on the analysis of two particularly interesting type III events.

\section{Instrument and observations}

Observations with an acousto-optic spectrometer (AOS) of the type Elson-E2 were made in Bleien (Switzerland) from February 15 to March 15, 2000 in the $1.0-2.0 \mathrm{GHz}$ range. A total number of 256 channels with a bandwidth of $1 \mathrm{MHz}$ and a channel separation of $3.9 \mathrm{MHz}$ were simultaneously observed. The integration and sampling times were equal and amounted to $40 \mathrm{~ms}$. The spectrometer was fed by a $7 \mathrm{~m}$ dish observing the full Sun.

A second spectrometer, the frequency-agile Phoenix2 instrument (Messmer et al. 1999), observed in parallel with the same antenna. Its frequency range was from 1.0 to $2.8 \mathrm{GHz}$ in 40 channels having a bandwidth of $10 \mathrm{MHz}$ each and a channel separation of $40 \mathrm{MHz}$. The integration time was $0.44 \mathrm{~ms}$, and the sampling time $20 \mathrm{~ms}$. The observations of Phoenix-2 were used to calibrate the AOS data in absolute time and in flux density.

The improvement by the AOS for individual measurements is a factor of 3.1, in agreement with the radiometer equation. Compared to the sample size of Phoenix-2 in frequency and time $(40 \mathrm{MHz} \times 20 \mathrm{~ms})$, the reduction in rms noise is a factor of 7.1.
The two spectrometers operated in parallel for about 250 hours. We searched for radio emission at the time of flares as given by the solar event list of NOAA (2000) and noted radio emission in the Phoenix-2 data during a total number of 14 solar flares. The bursts included 6 patches of continuum emission, 1 pulsation, two synchrotron emissions and 5 type III bursts. The registrations by the AOS added the following information:

- Every radio event of any kind showed type III emission in AOS data in addition. The only exception to this rule was one broadband radiation probably emitted by the gyro-synchrotron mechanism;

- Flares reported by NOAA without radio emission in the $1-2 \mathrm{GHz}$ band and not detected in Phoenix-2 data often were accompanied by short, narrowband type III emission in AOS data. This was not the case though for every small flare ( $\leq \mathrm{C}$-class $)$.

The first analysis of the data suggests as the main result of higher sensitivity the registration of a larger number of small type III bursts. The total number of such bursts in the AOS data is limited both by sensitivity as well as by possible confusion with interference. In some cases of short and narrowband bursts, it was not possible to recognize a drift in frequency and time, the characteristic for type III bursts. Without this signature, the classification of the burst type and even the identification as a solar event is not possible beyond doubt.

The frequency distribution in peak flux of decimetric type III bursts is not known. At kilometer wavelength Fitzenreiter et al. (1976) found a power-law distribution with an exponent between -1.2 and -1.7 . Aschwanden et al. (1998) find exponents in the same range at meter wavelengths. Assuming a power-law distribution with an exponent of -1.4 , a sensitivity increase by a factor 7 should result in a larger burst number in AOS data than Phoenix-2 by a factor of 2.2. The observed increase is a factor of 4.5. Several reasons may be responsible for this, including better burst recognition by enhanced frequency resolution, bias in the search procedure or a change in the distribution at low fluxes.

\section{Spikes and type III bursts}

Decimeter type III bursts observed at peak flux densities between 8 and 18 sfu are presented in Fig. 1. For the first time, we report such bursts occurring at higher frequencies than decimeter narrowband spikes. The spikes around $1200 \mathrm{MHz}$ were also recorded by the frequencyagile spectrometer, but the type III bursts become only marginally noticeable after appropriate integration. The temporal correlation between spike activity and type III bursts is not in detail. The two emissions are well separated in frequency at about $1400 \mathrm{MHz}$. The drift rates of the decimeter type III bursts are $+570 \pm 40 \mathrm{MHz} / \mathrm{s}$, thus reversed and in the range typical at the frequency (Meléndez et al. 1999). The positive sign indicates that 


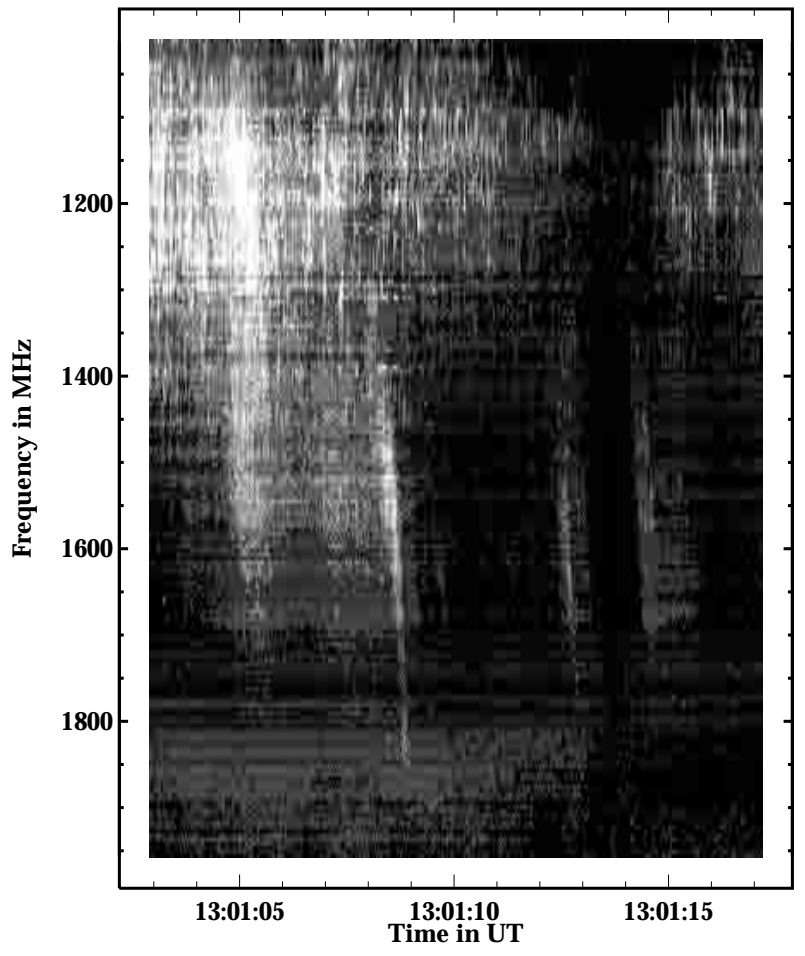

Fig. 1. Radio spectrogram recorded on 2000 February 22 by the AOS. At the top of the figure (low frequencies), narrowband spikes are visible up to about $1400 \mathrm{MHz}$. Between 1400 and $1800 \mathrm{MHz}$ at least 5 reversed drifting type III bursts are discernible

the radiating electron beams propagate to higher density, i.e. downward in the corona. Assuming that type III bursts and spikes are emitted at similar frequencies (as suggested by the close correlation of meterwave spikes and type III bursts, Benz et al. 1996), the observed type III emission most likely originates at lower altitude than the spike source. This makes it difficult to interpret the spike emission as originating from the footpoints of loops, contrary to the most favored scenario (cf. Sect. 1).

The Tremsdorf Observatory has recorded a faint regular-drift type III burst at 13:00:48 UT on $200 \mathrm{MHz}$. Even fainter type III-like activity occurred at 13:01:06 UT around $600 \mathrm{MHz}$. The San Vito radio flux meters report a peak value of only $60 \mathrm{sfu}$ at $245 \mathrm{MHz}$ (NOAA 2000). Thus the signatures of upward propagating electron beams are about one order of magnitude stronger than the emission of the downward beams. This is consistent with the previous non-detection of the latter.

We also note that the regular type III bursts (in the Tremsdorf data) and the reversed drift type IIIs (in the AOS data) are not symmetrical. This we interpret as evidence that the reversed-drifting structures are very likely not of instrumental origin (see below).

\section{New type of interference}

Figure 2 displays an unusual type III-like event. The overview observed with the frequency-agile spectrometer is shown in Fig. 2a. The burst apparent in Fig. 2b, recorded by the multi-channel instrument, is not visible in the overview. Simultaneously and later, decimeter pulsations have been observed by both spectrometers. Their low-sensitivity recording is shown in Fig. 2a. Pulsating continuum emission has recently been modeled by Kliem et al. (2000), suggesting repeated secondary reconnection of magnetic islands in a current sheet.

Figure $2 \mathrm{~b}$ shows slow-drifting emission, not visible in low-resolution (Fig. 2a). There is a distinct symmetry in frequency with an axis at $1392 \mathrm{MHz}$, forming a fish-shaped structure. The peak flux at the resolution of AOS was $62.5 \mathrm{sfu}$, but most of the upper branch is around $10 \mathrm{sfu}$. The rms noise is $1.2 \mathrm{sfu}$. The duration of individual bursts at intermediate frequencies is $\leq 1 \mathrm{~s}$.

The upper part of the event resembles a burst of subtype U. Type III bursts with U-shape (inverted in the representation of the frequency axis in Fig. 2b) are well known in the meter wavelength range and are generally interpreted as emissions of electron beams following magnetic loops in the corona (e.g. review by Benz 1993; Aurass \& Klein 1997). In the $1-2 \mathrm{GHz}$ range, U-bursts were observed with a total duration between $4-5$ s by Aschwanden et al. (1992). The inverse of a type U burst has never been reported, although a somewhat similar structure, called Y-bursts, has been noticed by Tarnstrom \& Zehntner (1975) and interpreted by magnetic reflection of the electron beam in a converging magnetic field. Also, rising branches after U-bursts have been reported by Caroubalos et al. (1987) who introduced the term "Nburst". Simultaneous U-bursts and Y-bursts have never been observed.

The fish-like structure in the spectrogram (Fig. 2b) is extremely symmetric and the up and down branches start within $0.1 \mathrm{~s}$. It may be suggestive of a simultaneous injection of bidirectional beams. Several geometries are possible including propagation into two similar magnetic islands (plasmoids). Thus a scenario is possible where acceleration by secondary tearing occurs in the center of an 8-shaped magnetic field as predicted by island formation in reconnecting current sheets.

In the following, we suggest that the fish-like structure in Fig. 2b is more likely caused by intermodulation of a solar radio burst with terrestrial interference. At the time of the emission feature, San Vito (NOAA 2000) reports an extremely strong radio burst of $10000 \mathrm{sfu}$ at $410 \mathrm{MHz}$. Considering the arbitrariness of the sampling frequency and the low time resolution (1 s) of the flux meter, the reported peak value must be a lower limit. At $606 \mathrm{MHz}$ RBR reports a peak flux of $45 \mathrm{sfu}$ (NOAA 2000).

Figure 3 displays the spectrogram of Tremsdorf observatory in the range $370-500 \mathrm{MHz}$. The data is not calibrated, and the background is not subtracted. At $400 \mathrm{MHz}$ the sensitivity of the instrument changes. A very intense U-burst dominates the picture.

Throughout the observations, strong terrestrial interference was observed by the AOS at $1827( \pm 4) \mathrm{MHz}$ (not shown in Fig. 2b, omitted by Phoenix-2). Even stronger interference is usually present at $995( \pm 4) \mathrm{MHz}$, well 


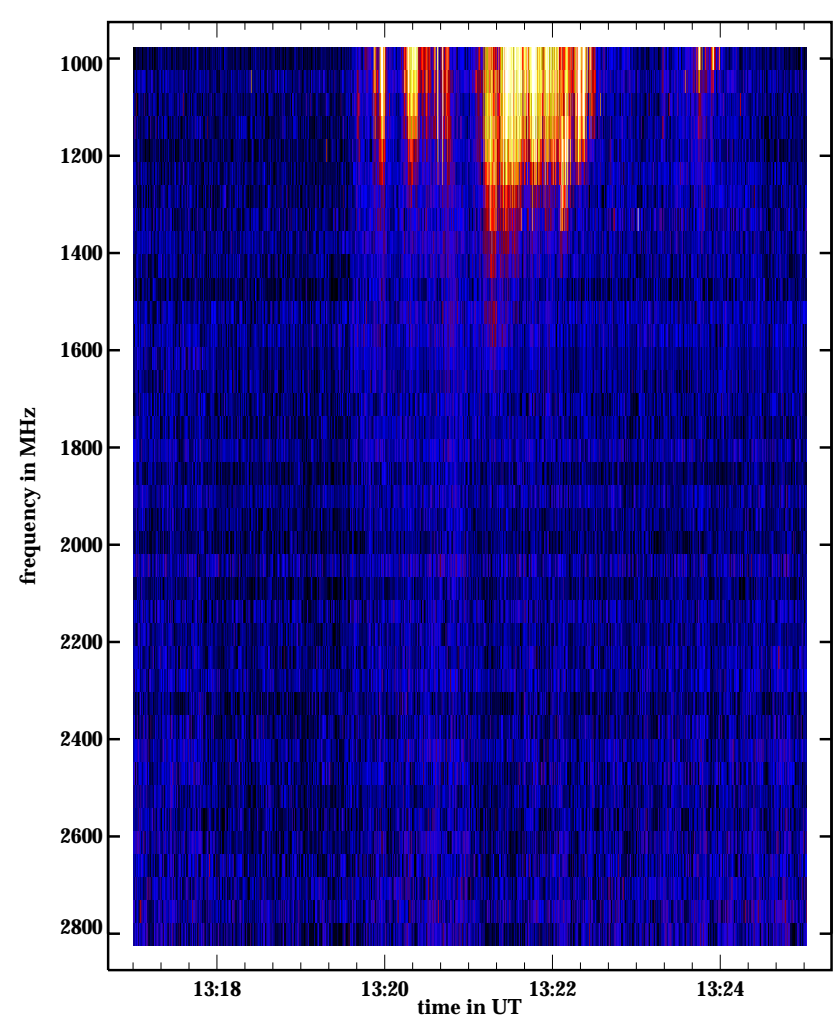

(a)

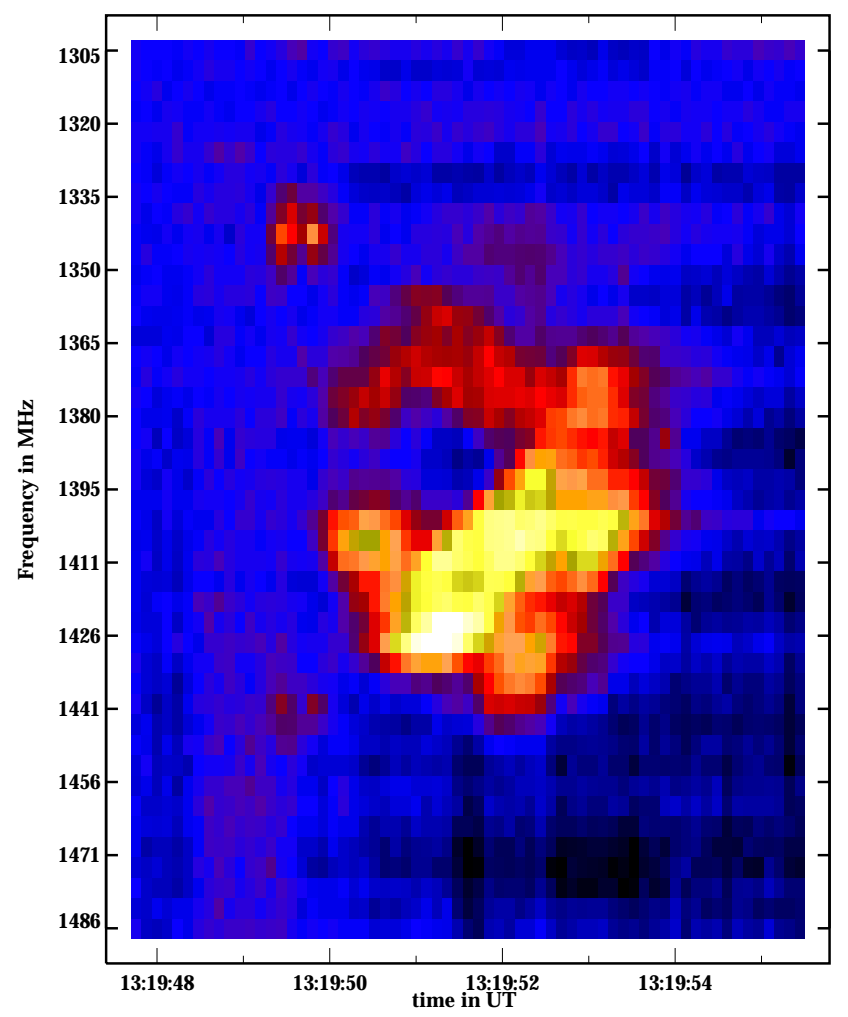

(b)

Fig. 2. a) Radio spectrogram recorded by the frequency-agile Phoenix-2, the standard instrument, giving an overview of the solar decimeter radiation in the $1-2.8 \mathrm{GHz}$ range. b) An enlargement of apparent type III activity as observed by the temporary multichannel receiver (AOS)

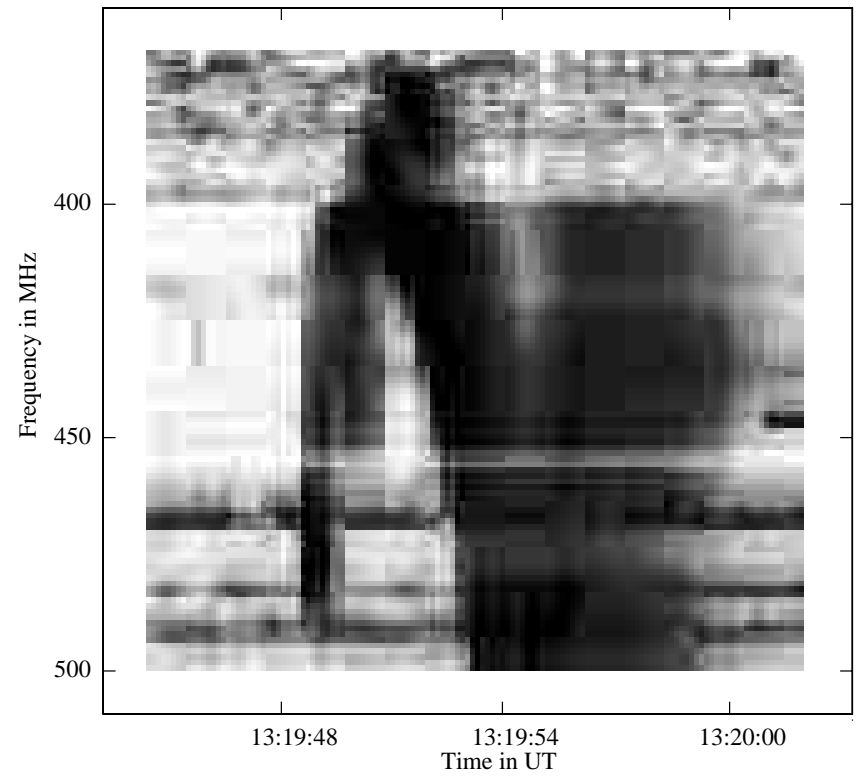

Fig. 3. Spectrogram observed by the Tremsdorf observatory (courtesy A. Klassen and G. Mann). Enhanced emission is shown dark

known from previous observations, but not observed in the present case. Both interferences are caused by transmitters for cellular phones. Intermodulation is possible by a non-linear element in the chain of propagation and reception (most likely an amplifier). It produces spurious signals at

$\omega=\omega_{\mathrm{i}} \pm \omega_{\mathrm{s}}$,

where $\omega$ is the observing frequency, $\omega_{\mathrm{i}}$ the frequency of the interference, and $\omega_{\mathrm{s}}$ the solar signal.

Equation (1) predicts two features in a spectrogram symmetric to the frequency of interference. Such structures cannot be perceived in the data of the frequencyagile spectrometer (Fig. 2a). The band of the AOS is such that it does not include both sides of the symmetric spurious patterns for neither of the two interferences.

Symmetric features can also appear in the presence of two interferences $\omega_{1}$ and $\omega_{2}$ if intermodulation occurs for $\omega_{1}+\omega_{\mathrm{s}}$ and $\omega_{2}-\omega_{\mathrm{s}}$. A symmetric structure $\omega_{0} \pm \omega_{\mathrm{s}}^{\star}$ may thus form, where the frequency of symmetry, $\omega_{0}$, is given by

$\omega_{0}=\frac{\omega_{1}+\omega_{2}}{2} \approx 1391( \pm 4) \mathrm{MHz}$

for the given interference frequencies and

$\omega_{\mathrm{s}}^{\star}=\omega_{\mathrm{s}}+\omega_{1}-\omega_{0}=\omega_{\mathrm{s}}-\omega_{2}+\omega_{0}$.

There is no steady emission at $\omega_{0}$. The spurious structure produced by the stronger interference appears more intense. These properties including the approximate value in Eq. (2) all agree well with the observed features in Fig. 2 b.

The agreement between the original image in the Tremsdorf spectrogram (Fig. 3) and its possible projections into the $1-2 \mathrm{GHz}$ band of the AOS by interferences 
at 955 and $1827 \mathrm{MHz}$ is reasonably good. The original Uburst has a range from $370 \mathrm{MHz}$ to $420 \mathrm{MHz}$ at the time of turnover. The predicted values for intermodulation with the $1827 \mathrm{MHz}$ interference, 1407 and $1457 \mathrm{MHz}$ respectively, include the observed range from 1407 to $1442 \mathrm{MHz}$. Intermodulation at $955 \mathrm{MHz}$ would cause features between 1325 and $1375 \mathrm{MHz}$, while the observed extremes are 1353 and $1373 \mathrm{MHz}$, respectively.

The timing is more difficult to compare, as the U-burst in the Tremsdorf spectrogram is followed by a weak continuum (possibly to be classified as a decimetric type $\mathrm{V}$ burst), and the timing accuracy and resolution of the Tremsdorf image are about $0.3 \mathrm{~s}$. Nevertheless, the timing agrees within the uncertainties, and the total duration of the U-burst of $5.5 \mathrm{~s}$ well contains the fish-like structure in the AOS data lasting $4.5 \mathrm{~s}$.

Therefore, the very likely interpretation of the apparent bidirectional U-burst in the AOS data (Fig. 2b) is a spurious signal produced by intermodulation. The most intense part of a strong U-burst is mirrored at two frequencies of strong interference and shifted up in frequency to $1.4 \mathrm{GHz}$. The flux density of the intermodulation pattern is at least 3 orders of magnitude weaker than the original solar signal.

The identification with intermodulation reveals a serious threat of high-sensitivity broadband spectroscopy by strong terrestrial interference. For a reliable detection of a weak signal at decimeter waves, the possibility of a mirror image of a meterwave burst must be excluded. This puts some doubts for example on bidirectional type III bursts recently reported in the $1-2 \mathrm{GHz}$ range (Ning et al. 2000, Fig. 1) spreading out symmetrically from a frequency of $1720 \mathrm{MHz}$ obviously interfered.

\section{Conclusions}

Enhanced sensitivity in the decimeter range greatly enlarges the number of detected type III bursts. As these bursts have a relatively small bandwidth and duration, high resolution in frequency and time is equally important. Spurious signals from intermodulation may be avoided technically i.e. by better amplifiers or excluded by monitoring the meter wavelength range of solar bursts.

The bursts trace out paths of unstable electron beams propagating away from the acceleration region. One month of observations has immediately produced exciting results, including the first case of reversed-drift type III bursts starting at the frequencies of a group of narrowband decimeter spikes. This observation puts doubts on the standard model of decimetric spikes occurring in the lowest parts of loops, but needs confirmation by similar cases.

More high-sensitivity observations are necessary for a complete overview on the activity of weak decimeter type III bursts. A systematic high-sensitivity survey in the frequency range $1-3 \mathrm{GHz}$, possibly combined with spatial resolution, have the potential to generate a clearer understanding of magnetic fields associated with the acceleration region of electrons in flares.

Acknowledgements. We thank Michael Arnold (ETH Zurich) and Max Wüthrich (University of Bern) for help in the realization of the temporary set-up. The acousto-optic spectrometer was kindly lent by the Microwave Department of the Institute of Applied Physics, University of Bern. Observations of the Tremsdorf spectrometer were essential in identifying the cause of the intermodulation pattern. They were kindly provided by A. Klassen and G. Mann. The work at ETH Zurich is financially supported by the Swiss National Science Foundation (grant No. 2000-061559.00).

\section{References}

Aschwanden, M. J., Bastian, T. S., Benz, A. O., \& Brosius, J. W. 1992, ApJ, 391, 380

Aschwanden, M. J., Dennis, B. R., \& Benz, A. O. 1998, ApJ, 497, 972

Aurass, H., \& Klein, K.-L. 1997, A\&AS, 123, 279

Benz, A. O. 1993, Plasma astrophysics: Kinetic processes in solar and stellar coronae (Dordrecht: Kluwer)

Benz, A. O. 1986, Solar Phys., 104, 99

Benz, A. O., Jaeggi, M., \& Zlobec, P. 1982, A\&A, 109, 305

Benz, A. O., Bernold, T. E. X., \& Dennis, B. R. 1983, ApJ, 271,355

Benz, A. O., Csillaghy, A., \& Aschwanden, M. J. 1996, A\&A, 309, 291

Benz, A. O., \& Güdel, M. 1987, Solar Phys., 111, 175

Benz, A. O., \& Kane, S. R. 1986, Solar Phys., 104, 179

Caroubalos, C., Poquerusse, M., Bougeret, J.-L., \& Crepel, R. 1987, ApJ, 319, 503

Conway, A. J., \& Willes, A. 2000, A\&A, 355, 751

Fitzenreiter, R. J., Fainberg, J., \& Bundy, R. B. 1976, Solar Phys., 46, 465

Kliem, B., Karlický, M., \& Benz, A. O. 2000, A\&A, 360, 715

Güdel, M., Benz, A. O., \& Aschwanden, M. J. 1991, A\&A, 251, 285

Meléndez, J. L., Sawant, H. S., Fernandes, F. C. R., \& Benz, A. O. 1999, Solar Phys., 187, 77

Melrose, D. B., \& Dulk, G. A. 1982, ApJ, 259, 844

Messmer, P., Benz, A. O., \& Monstein, C. 1999, Solar Phys, 187,335

Miller, J. A., Cargill, P. J., Emslie, A. G., et al. 1997, JGR, 102,14631

Ning, Z., Fu, Q., \& Lu, Q. 2000, Solar Phys., 194, 137

NOAA, U. S., Department of Commerce, Space Environment Center 2000, gopher://solar.sec.noaa.gov:70/00/lists/radio/rad

Priest, E. R., \& Forbes, T. 2000, Magnetic Reconnection, MHD Theory and Applications (Cambridge University Press, Cambridge, UK)

Sharma, R. R., Vlahos, L., \& Papadopoulos, K. 1982, A\&A, 112,377

Simnett, G. M., \& Benz, A. O. 1986, A\&A, 165, 227

Staehli, M., \& Benz, A. O. 1987, A\&A, 175, 271

Robinson, P. A., \& Benz, A. O. 2000, Solar Phys., 194, 371

Tandberg-Hanssen, E., \& Emslie, A. G. 1988, The Physics of Solar Flares (Cambridge University Press)

Tarnstrom, G. L., \& Zehntner, Ch. 1975, Nature, 258, 693 